\title{
Monitoring and Controlling in an Industrial Service Ecosystem
}

\author{
Marco Taisch, Mohammadreza Heydari Alamdari, and Christian Zanetti \\ Politecnico di Milano, Piazza Leonardo Da Vinci 32, 20133, Milan, Italy \\ \{marco.taisch, mohammadreza.heydari, critiano. zanetti\} @polimi.it
}

\begin{abstract}
Industrial companies usually have a defined product development process, but they lack a sufficiently defined service development process as found in traditional service companies [1]. Many firms recognize that the existing corporate structures and processes do not allow for efficient development and market positioning of innovative services. In addition, they are faced with the problem of being poorly equipped with appropriate approaches, methodologies and tools for an efficient development of services. Other problems can include the high complexity of services, missing organizational structures, lack of innovative climate and inadequate qualifications [1], [2]. It should be emphasized at this point that the lack of service performance assessment and monitoring is the cause of errors, redundancy and duplication of work between the main actors of industrial service ecosystem [3].

In this respect, the purpose of this paper is therefore to lay the foundations for a monitoring and controlling framework related to an industrial service ecosystem. These foundations are based on establishing methods and tools to select, identify and use proper KPIs within an industrial service ecosystem. Accordingly, monitoring framework will support enterprises to control their goals and decision-making processes within the industrial service ecosystem while providing them a monitoring cockpit.
\end{abstract}

Keywords: Monitoring Framework, Industrial Service Ecosystem \& Key Performance Indicators.

\section{Introduction}

In the present economic climate, the service sector is becoming increasingly important for developed economies generally. In the European Union the service sector creates about three quarters of the overall Gross Domestic Product (GDP) [4] and has high impact on the European labor market, as almost three-quarters of all European jobs are allocated here [4]. Moreover, firms - industrial companies in particular - are striving to become more service-oriented in order to meet users' expectations and customer's requirements [2].

As far as the creation of high value services in industrial ecosystem context through methods for monitoring and controlling the service ecosystem is concerned, all actors in the industrial service ecosystem need to learn how to combine their complementary core competencies across sectors, and how to share highly specialized 
knowledge about new functions, features, and processing procedures [5]. Also problems like bureaucracy, inefficiency or missing process quality are tackled by functional modules that are encapsulated by business as well as IT services. Therefore, ecosystem monitoring and governance can be used as a set of guidelines to adapt, coordinate and safeguard autonomous action performed by different actors, collectively working on a joint plan determined by collaboration, where risks, resources, responsibilities and rewards are shared to achieve a common goal.

Furthermore, Service management needs to be able to measure and control the service system in order to ensure maximum productivity. By using monitoring and controlling framework we will be able to elaborate the framework as a useful method to help the ecosystem to evaluate their service systems. Apart from analyzing the service system, the framework will provide, collect and share the results of the controlling activities among service ecosystem members.

Monitoring and controlling framework will allow regular observation and recording of activities, controlling and measuring actual performances and presenting a visualization system which can guide the industrial service ecosystem on taking corrective actions. The results of the monitoring framework, then, can be fundamental for decision making. Likewise, monitoring and controlling framework will be able to give a method of assessment to optimize the resources and support decision making. This will be useful to provide the partners with effective solutions for governance.

Finally, we will propose the monitoring framework by means of visualization method to visualize the data as a guiding strategic tool. This framework will be integrated into the service ecosystem platform to support service management, exchange and evaluation within the manufacturing networks.

The next section will discuss and analyze different methods and frameworks. Relevant connections between monitoring and service activities will also be highlighted and finally, a conceptual schema for monitoring framework will be proposed.

\section{Relevant Monitoring and Controlling Methods}

In this section some relevant existing control systems which support the decision making within the industrial service ecosystem will be analyzed. A few tools (recommendations, models, methods, systems and frameworks), proposed by various researchers and practitioners to control and monitor performances, are going to be studied. This paper, then, will focus on defining the specific components and elements necessary to control and monitor. Each methodology has its own criteria, so in order to align with the objectives of the paper, we will try to select and extract just the components and elements which are important to monitor and evaluate the industrial service ecosystem within its internal departments and the ecosystem network by integrating them with ICT tools.

As mentioned in the last section the purpose of this paper is to lay down the foundations of monitoring framework, by using the world of frameworks, methodologies and tool boxes which can support various levels of performance indicators in the industrial service ecosystem. So, according to aforementioned importance we will focus on the three extracted models for the classification of industrial service ecosystem activities. 


\subsection{GRAI (Graph with Results and Activities Interrelated) Model}

The GRAI method is an enterprise modeling method that can represent and analyze the operations of all or part of a production activity. The strength of the GRAI method lies in its ability to enable modelers to effectively model the decision-making system of the company, i.e. organizational processes that generate decisions [6].

Meanwhile, the GRAI model describes the control of a variety of organizations using a combination of system theory, control theory and particularly hierarchical theory. Indeed, even if each project task is clearly defined, the main difficulty concerns its management: what are the required decisions to take periodically to manage tasks? The GRAI decisional Grid aims to define the "set of decisions" from the strategic level (where the horizon is longer than the project duration in order to take into account the dissemination) to the tactical and operational levels [7]. This model defines a reference model of decisions for project management. It defines also periods in a horizon: the periods determine the timing to check the results.

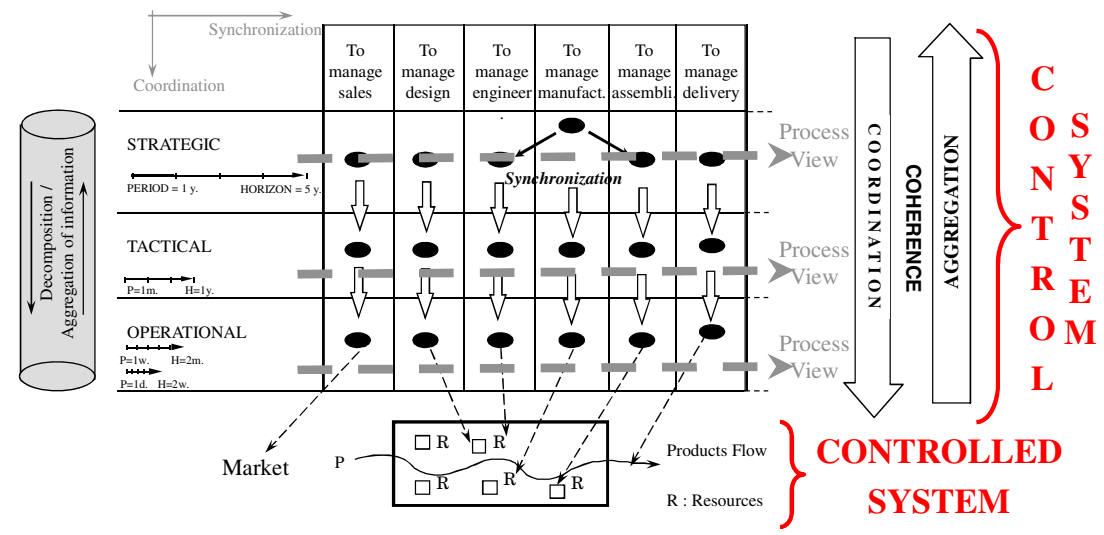

Fig. 1. GRAI conceptual model [8]

The Method is composed of three main building blocks. The first one is the reference model, called GRAI model, which is a consistent set of concepts that models any production system. Therefore it is clearly independent of the case upon which the method is applied. The second building block is concerned by graphical modeling languages that enable to instantiate the concepts of the GRAI model to build the specific model of the studied case. Finally, the third is a structured and participative approach in which actors and steps are defined. The purpose of such an approach is to act as effectively as possible and to save time. The interest of a conceptual model is to relate the various concepts in order to show their coherence, to avoid redundancies and to have a complete model. Generally, in the GRAI model for typical enterprise, six functions are taken into account: to manage production, to manage sales, to manage design, to manage development, to manage production, to manage assembling, and to manage delivery.

By synthesizing the GRAI model with the next two methods which will be explained in next sections, we will try to make a hierarchical decomposition to facilitate the integration between decisional levels and between functions. 


\subsection{The Model Driven Service Engineering Architecture (MDSEA)}

The proposed MDSEA framework defines KPIs for the monitoring of the service ecosystem and analyses the KPIs in terms of implementation. KPIs can be separated into three components: IT, Organization and Human resources. The criteria of these performance indicators will be related to traditional performances such as cost, quality, lead time and efficiency, as well as other kinds of performances such as interoperability, flexibility, environment, etc.

By defining the framework for service ecosystem modeling around three abstraction levels which will be defined below, we will aim to accomplish this framework by dividing the KPIs into three separate components: Business Service Modeling (BSM), Technology Independent Modeling (TIM), and Technology Specific Modeling (TSM) [9]. The mentioned components are important for forming the foundation to monitoring the performance indicators within the ecosystem. In the section below, a brief definition of mentioned components are considered:

- Business Service Modeling (BSM): specifies the models at the global level, describing the running of the enterprise or set of enterprises as well as the links between these enterprises.

- Technology Independent Modeling (TIM): is the model at a second level of abstraction, independent from the technology used to implement the system.

- Technology Specific Modeling (TSM): combines the specification in the TIM model with details that specify how the system uses a particular type of technology (such as, for example, IT platform, Machine technology organization structure or human profile).

So, based on the decomposition above (BSM, TIM \& TSM) it is necessary to ensure that at each level of decomposition, performance evaluation is possible. The framework of the performance indicators is shown in figure 2 , below.

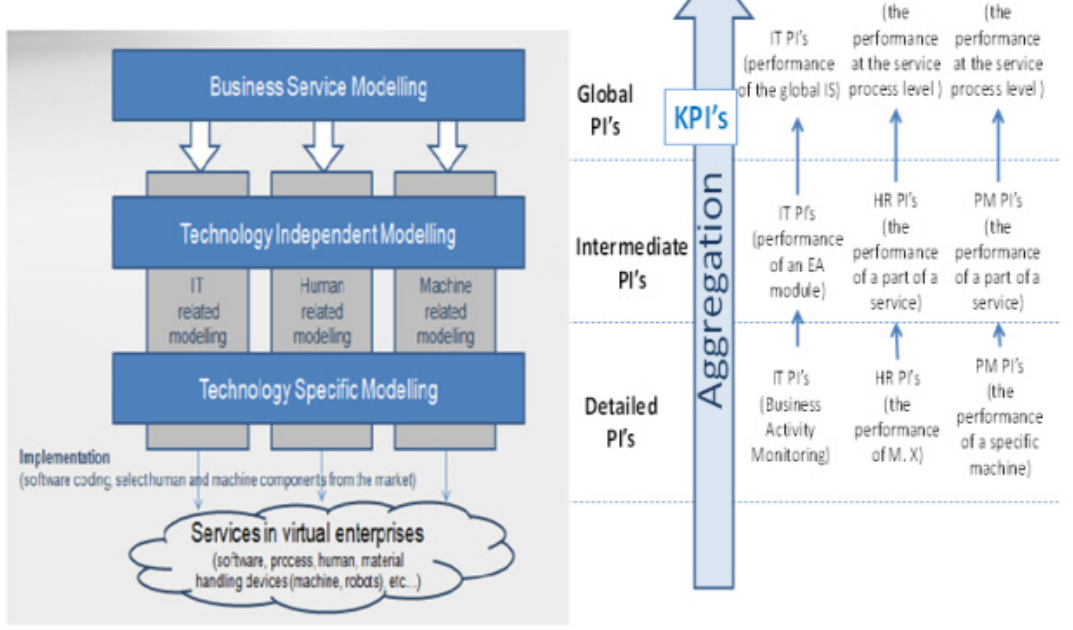

Fig. 2. Performance Indicator in the frame of MDSEA 
MDSE Architecture and GRAI Model have been chosen on the basis of the decomposition by level of decision and decomposition by abstraction level. On the other hand the next section will explain Unified Governance Framework (UGF), which will focus on aiding enterprises in implementing consistent governance with a certain focus on the use of ICT and ICT-based services.

\subsection{Unified Governance Framework}

This section aims to explain Unified Governance Framework (UGF) model and its related components by highlighting their advantages and synthesizing the main issues with previously mentioned methods.

IBMs Unified Governance Framework (UGF) [10] is intended to cover the entire field of enterprise governance, with a focus on how IT-related services and components can support governance. The main purpose and novelty of UGF is to serve as a framework also for the governance-enabling technology for the overall enterprise governance field. In the diagram of the UGF below, the inner three layers are those of an enterprise, while the outer (red) layers represent the environment.

The core of UGF is the highest-level components, which are shown in figure below. A component model is a grouping of related functions and capabilities into components that communicate through relatively well-defined interfaces. A component can contain organizational, structures, processes, people, and technology. The specific purpose of UGF is to focus on enterprise governance, i.e., to distinguish and describe governance components in more depth than the rest of the enterprise. Three specific layers are described on the following figure and they may be referred to in the service management sector as well as in the organization as a whole.

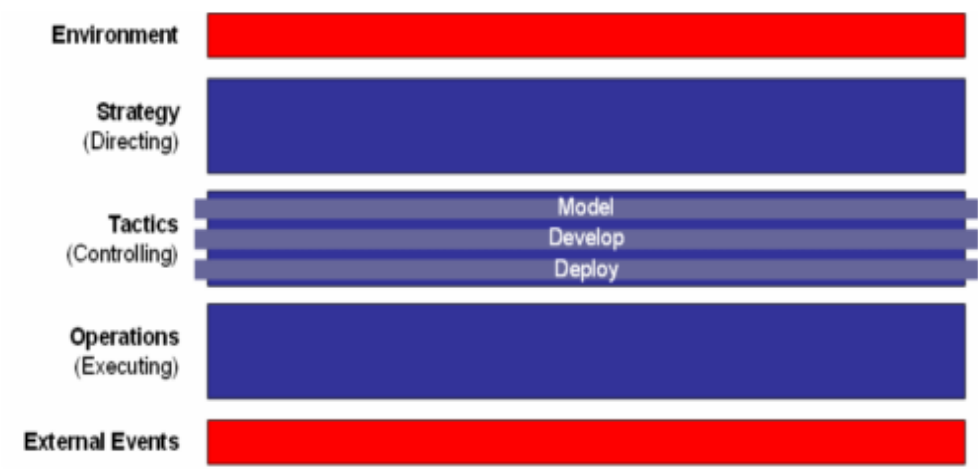

Fig. 3. - UGF layers

In the strategy layer, the normal enterprise capabilities are summarized. Governance deals with three main aspects: business performance goals, legal issues, and risk. In the strategy layer, aspects involve overall analysis, goal setting, and establishment of appropriate organizational structures, such as service performance goals and measurements. 
In the tactical layer, the normal enterprise capabilities are defined in terms of process and information management, and resource management. The former corresponds to business parts organized by lines of business and business processes, the latter to functional units like IT, HR (human resources), and facilities. Each of the component's performance management, controls management and risk management, correspond to the strategy component above it. In the operation layer, the normal enterprise capabilities are evaluated in a similar way and they are based on day-to-day tasks.

Finally, with reference to the UGF model's core components, GRAI decomposition model by level of decisions, and MDSE Architecture by level of abstraction; we aim to generate a new service monitoring framework by synthesizing the three models mentioned above.

\section{Conceptual Schema for Monitoring Framework (Proposition)}

In this section, we present a proposition of a conceptual schema of monitoring framework in which GRAI and MDSEA models have been synthetized with UGF model in order to create a conceptual monitoring framework. Conceptual monitoring framework will be developed to support service ecosystem in order to achieve the following goals:

- To support the service performance assessment to monitor performances and prevent errors causing and redundancy or duplication of work among the main actors of service ecosystem;

- Facilitate the integration between decisional levels \& functions.

Relevant methods, which are useful to generate and control service monitoring, have been studied in this research. All the requirements need to be separated into the three levels of decomposition (strategic, tactical and operational) on the basis of the MDSE Architecture in order to classify quantity and quality KPIs inside one unique model.

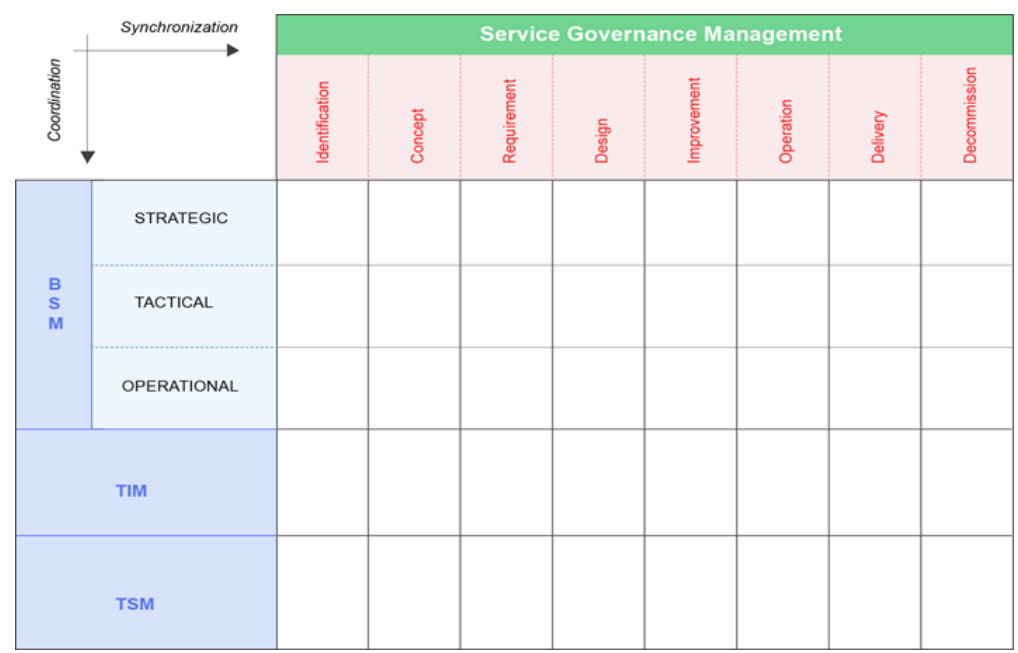

Fig. 4. Conceptual schema for monitoring framework (proposition) 
Relevant PIs and KPIs can be then generated on the basis of the requirements identified inside the monitoring framework. They can be presented in three levels, as following the decomposition of BSM level (i.e. Strategic, Tactical and Operational). The remaining KPIs which are collected in TIM and TSM levels specify the parameters which can be used as a supporting means with reference to technology implementation. The MDSE Architecture has been used, therefore, as a filter for KPIs, in order to define in detail which parameter is affecting which functions inside the SLM (Service Lifecycle Management) of a service system and at which level within the enterprise environment.

As it is possible to notice from our proposition of the Conceptual schema for monitoring framework, several phases have been identified inside the Service monitoring management axis. These phases have to be considered like the "functions" which have been identified and used in the GRAI method. These functions have been identified following the service life cycle phases. Service engineering components can cover the whole service lifecycle phases from early stages, where both intrinsic and extrinsic parameters and requirements should be defined and measured, to the final decommissioning steps, which could give rise to further business opportunities (recycling, refurbishing, different use, etc.).

As it has been discussed above, the functions can be simplified in order to set the assessment just during particular phases. As far as the SLM is concerned, we can consider upper phases related to Service ecosystem (identification, concept, requirement) and lower phases to manufacturing enterprises (design, implementation, operation, decommission). The conceptual schema for monitoring framework tries to synthesize MDSE Architecture with the modeling of a service system along the SLM lifecycle. BSM aims at elaborating high abstraction level model from business users' point of view. TIM gives service system specifications independent of technology for implementation. TSM adds necessary technology specific information related to implementation options. The aim of this framework is to help end users represent and describe the intended service and its system from various points of view, and give structure in order to help the decision making and the controlling activities.

\section{Conclusion}

In this paper a monitoring methodology has been proposed in order to support and define a clear monitoring framework to be used by industrial service ecosystem. Several methods have been studied and synthesized in order to create a monitoring framework for service: GRAI method, UGF and MDSEA.

It should be emphasized that the proposed conceptual framework can represent a clear and open structure to share knowledge and resources among the partners inside the industrial service ecosystem. Therefore, the framework needs to integrate within the service ecosystem platform so as to support service management, exchange and evaluation within the manufacturing networks. The framework can also be used as a supporting toolset to generate specific KPIs related to end users' core activities.

Further steps are needed in order to validate the mentioned framework within a real condition in an industrial service ecosystem. 


\section{References}

[1] Bullinger, H.-J.M.: Service Engineering - Entwicklung und Gestaltung von Dienstleistungen. In: Bruhn, M., Meffert, H. (eds.) Handbuch Dienstleistungs management. Von der, 2nd edn., Wiesbaden, pp. 149-175 (2001)

[2] Freitag, H.: Development and Management international services. Study on the export of services for German companies. Fraunhofer IRB Verlag, Stuttgart (2007)

[3] Bullinger, H.-J., Scheer, A.-W.A.: Service Engineering: Entwicklung und Gestaltung innovativer Dienstleistungen. Springer (2003)

[4] Wto, T.: International Trade Statistics. WTO Publications (2008)

[5] Senge, P.M., Carstedt, G.: Innovating Our Way to the Next Industrial Revolution. In: Leibold, G.P.M. (ed.) Strategic Management in the Knowledge Economy: New Approaches and Business Applications, pp. 24-44. Publicis Corporate Publishing \& WileyVCH-Verlag, Erlangen (2002)

[6] Doumeingts, G., Vallespir, B.: Decisional modelling GRAI grid. In: Bernus, K.M.P. (ed.) International Handbook on Information Systems. Springer, Berlin (1998)

[7] Doumeingts, G., Yves, D.: Production management and enterprise modelling, Computers in Industry. Elsevier (2000)

[8] Doumeingts, G., Yves, D.: Enterprise modeling techniques to improve efficiency of enterprises. International Journal of Production Planing \& Control, 146-163 (2001)

[9] OMG. Unified Modeling Language TM (OMG UML), Infrastructure -version2.4.1 (2011), http: / / www. omg .org/spec/UML/2 .4 .1/Infrastructure/PDF /

[10] Birgit Pfitzmann, C.P.: IBM's Unified Governance Framework (UGF) Initiative. IBM Software Group, Zurich (2007) 